\title{
LONG-RUN AVAILABILITY OF A PRIORITY SYSTEM: A NUMERICAL APPROACH
}

\author{
EDMOND J. VANDERPERRE AND STANISLAV S. MAKHANOV
}

Received 29 June 2004

We consider a two-unit cold standby system attended by two repairmen and subjected to a priority rule. In order to describe the random behavior of the twin system, we employ a stochastic process endowed with state probability functions satisfying coupled Hokstadtype differential equations. An explicit evaluation of the exact solution is in general quite intricate. Therefore, we propose a numerical solution of the equations. Finally, particular but important repair time distributions are involved to analyze the long-run availability of the T-system. Numerical results are illustrated by adequate computer-plotted graphs.

\section{Introduction}

Standby provides a powerful tool to enhance the reliability, availability, quality, and safety of operational plants, see, for example, $[3,7,14]$. However, in practice, standby systems are often subjected to an appropriate priority rule. For instance, the external power supply station of a technical plant has usually overall priority in operation with regard to an internal (local) power generator kept in cold or warm standby [3]. The local generator is only deployed if the external power station is down.

Cold or warm standby systems subjected to a priority rule and attended by a repair facility have received considerable attention in the current literature, see, for example, $[1,2,4,5,8,9,10,11,12,13,16,17,18,19,20,21]$.

As a variant, we consider a twin system composed of a priority unit (the p-unit) and a nonpriority unit (the $\mathbf{n}$-unit) kept in cold standby. The $\mathbf{p}$-unit has overall (break-in) priority in operation with regard to the $\mathbf{n}$-unit, that is, the $\mathbf{n}$-unit is only used when the p-unit is down. In order to avoid undesirable delays in repairing failed units, we suppose that the entire system (henceforth called the T-system) is attended by two different repairmen. The T-system satisfies the usual conditions, that is, independent identically distributed random variables, instantaneous and perfect switch [3], and perfect repair [6]. Each repairman has his own particular task. Repairman $\mathcal{N}$ is skilled in repairing the $\mathbf{n}$-unit, whereas repairman $\mathscr{P}$ is an expert in repairing the $\mathbf{p}$-unit. Both repairmen are jointly busy, if and only if, both units (p-unit and $\mathbf{n}$-unit) are down. In any other case, at least one repairman is idle. 
In order to describe the random behavior of the T-system, we employ a stochastic process endowed with transition probability functions satisfying steady-state Hokstadtype differential equations. Unfortunately, the exact solution procedure is quite intricate (see, [21, page 359] and Remark 4.1). Therefore, we propose a numerical solution of the equations.

Finally, current repair time distributions (such as the Weibull-Gnedenko distribution) are involved to compute the long-run availability of the $\mathbf{T}$-system. The results are illustrated by adequate computer-plotted graphs.

\section{Formulation}

Consider a $\mathbf{T}$-system satisfying the usual conditions. The $\mathbf{p}$-unit has a constant failure rate [15] $\lambda>0$ and a general repair time distribution $R(\cdot), R(0)=0$, with mean $\rho$. The operative $\mathbf{n}$-unit has a constant failure rate $\lambda_{s}>0$, but a zero failure rate in standby (the so-called cold standby state) and a general repair time distribution $R_{S}(\cdot), R_{S}(0)=0$, with mean $\rho_{s}$. In order to describe the random behavior of the $\mathbf{T}$-system, we introduce a stochastic process $\left\{N_{t}, t \geq 0\right\}$ with arbitrary discrete state space $\{A, B, C, D\} \subset[0, \infty)$, characterized by the following mutually exclusive events:

(i) $\left\{N_{t}=A\right\}$ : "the $\mathbf{p}$-unit is operative and the $\mathbf{n}$-unit is in cold standby at time $t$,"

(ii) $\left\{N_{t}=B\right\}$ : "the $\mathbf{n}$-unit is operative and the $\mathbf{p}$-unit is under repair at time $t$,"

(iii) $\left\{N_{t}=C\right\}$ : "the $\mathbf{p}$-unit is operative and the $\mathbf{n}$-unit is under repair at time $t$,"

(iv) $\left\{N_{t}=D\right\}$ : "both units are simultaneously down at time $t$."

State $D$ is called the system-down state.

Figures $2.1,2.2,2.3$, and 2.4 display a functional block diagram of the T-system operating in states $A, B, C$, and $D$.

Observe that the process $\left\{N_{t}, t \geq 0\right\}$ is non-Markovian. A Markov characterization of the process is piecewise and conditionally defined by:

(i) $\left\{N_{t}\right\}$, if $N_{t}=A$ (i.e., if the event $\left\{N_{t}=A\right\}$ occurs),

(ii) $\left\{\left(N_{t}, X_{t}\right)\right\}$, if $N_{t}=B$, where $X_{t}$ denotes the remaining repair time of the $\mathbf{p}$-unit under progressive repair at time $t$,

(iii) $\left\{\left(N_{t}, Y_{t}\right)\right\}$, if $N_{t}=C$, where $Y_{t}$ denotes the remaining repair time of the $\mathbf{n}$-unit under progressive repair at time $t$,

(iv) $\left\{\left(N_{t}, X_{t}, Y_{t}\right)\right\}$, if $N_{t}=D$.

The state space of the underlying piecewise linear (vector) Markov process is given by

$$
A \cup\{(B, x) ; x \geq 0\} \cup\{(C, y) ; y \geq 0\} \cup\{(D, x, y) ; x \geq 0 ; y \geq 0\}
$$

Next, we consider the T-system in stationary state (the so-called ergodic state) with invariant measure $\left\{p_{K} ; K=A, B, C, D\right\}, \sum_{K} p_{K}=1$, where

$$
p_{K}:=\lim _{t \rightarrow \infty} \mathbf{P}\left\{N_{t}=K \mid N_{0}=A\right\}
$$




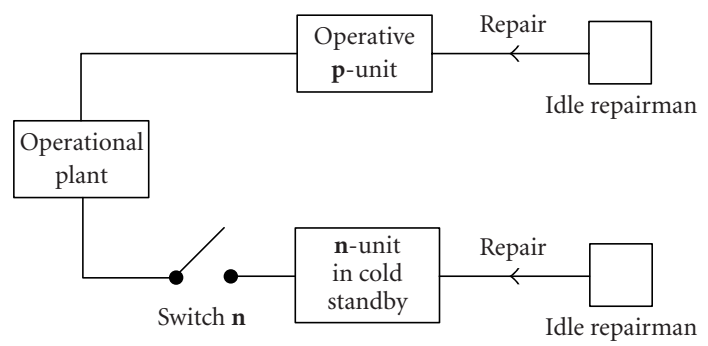

Figure 2.1. Functional block diagram of the T-system operating in state $A$.

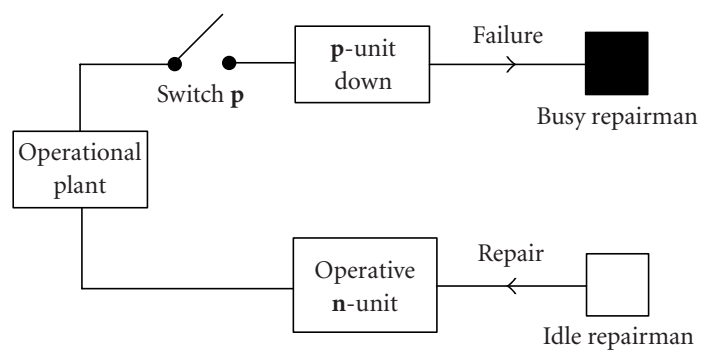

Figure 2.2. Functional block diagram of the $\mathbf{T}$-system operating in state $B$.

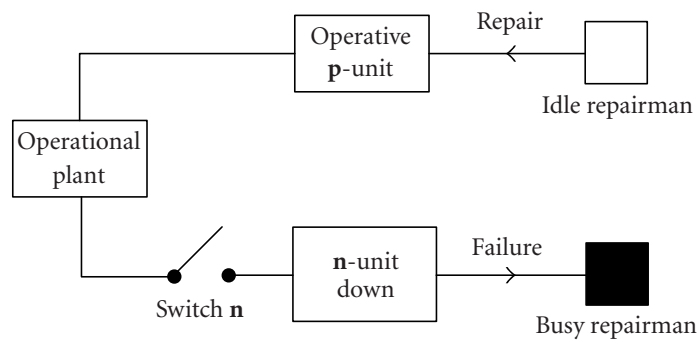

Figure 2.3. Functional block diagram of the $\mathbf{T}$-system operating in state $C$.

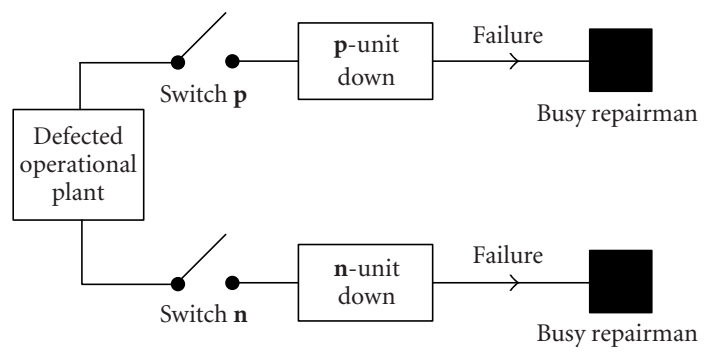

Figure 2.4. Functional block diagram of the T-system in state $D$. 
It can be demonstrated that the invariant measure exists for arbitrary $R$ and $R_{S}$ with finite mean. However, in order to keep the analysis as simple as possible, we henceforth assume that $R$ and $R_{S}$ have bounded densities on [0, ), denoted by $r$ and $r_{s}$. Finally, we introduce the measures

$$
\begin{gathered}
p_{B}(x) d x:=\lim _{t \rightarrow \infty} \mathbf{P}\left\{N_{t}=B, X_{t} \in(x, x+d x] \mid N_{0}=A\right\}, \\
p_{C}(y) d y:=\lim _{t \rightarrow \infty} \mathbf{P}\left\{N_{t}=C, Y_{t} \in(y, y+d y] \mid N_{0}=A\right\}, \\
p_{D}(x, y) d x d y:=\lim _{t \rightarrow \infty} \mathbf{P}\left\{N_{t}=D, X_{t} \in(x, x+d x], Y_{t} \in(y, y+d y] \mid N_{0}=A\right\} .
\end{gathered}
$$

Note that, for instance, $p_{D}=\int_{0}^{\infty} \int_{0}^{\infty} p_{D}(x, y) d x d y$.

\section{Long-run availability}

We recall that the T-system is only available (functioning) in states $A, B$, and $C$. Therefore, the long-run availability of the operational plant, denoted by $\mathscr{A}$, is given by $\mathscr{A}=1-p_{D}$. Invoking the substitutions $p_{B}(\cdot)=p_{A} \varphi_{B}(\cdot), p_{C}(\cdot)=p_{A} \varphi_{C}(\cdot), p_{D}(\cdot, \cdot)=p_{A} \varphi_{D}(\cdot, \cdot)$ and the law $\sum_{K} p_{K}=1$ entails that $p_{A}=1 /\left(1+\Phi_{B}+\Phi_{C}+\Phi_{D}\right)$, where $\Phi_{B}:=\int_{0}^{\infty} \varphi_{B}(x) d x$, $\Phi_{C}:=\int_{0}^{\infty} \varphi_{C}(y) d y$ and $\Phi_{D}:=\int_{0}^{\infty} \int_{0}^{\infty} \varphi_{D}(x, y) d x d y$. Hence,

$$
\mathscr{A}=\frac{1+\Phi_{B}+\Phi_{C}}{1+\Phi_{B}+\Phi_{C}+\Phi_{D}} .
$$

\section{Differential equations}

In order to determine the $\varphi$-functions, we first construct a system of coupled steady statetype differential equations based on a time-independent version of Hokstad's supplementary variable technique (see, e.g., [22, page 526] for further details). For $x>0, y>0$, we obtain

$$
\begin{gathered}
\lambda=\varphi_{B}(0)+\varphi_{C}(0), \\
\left(\lambda_{s}-\frac{d}{d x}\right) \varphi_{B}(x)=\varphi_{D}(x, 0)+\lambda r(x), \\
\left(\lambda-\frac{d}{d y}\right) \varphi_{C}(y)=\varphi_{D}(0, y), \\
\left(-\frac{\partial}{\partial x}-\frac{\partial}{\partial y}\right) \varphi_{D}(x, y)=\lambda_{s} \varphi_{B}(x) r_{s}(y)+\lambda \varphi_{C}(y) r(x) .
\end{gathered}
$$

Remark 4.1. A particular but important family $\mathscr{F}$ of current repair time distributions with nonrational characteristic functions, such as the Weibull-Gnedenko and Lognormal distributions, are fairly suitable to model repair times. Unfortunately, if both $R$ and $R_{S}$ belong to $\mathscr{F}$, an explicit evaluation of the exact solution of (4.1), (4.2), (4.3), and (4.4) in terms of finite linear combinations of known algebraic and/or transcendental functions is as good as excluded (see [21, page 361] for further details). Therefore, we propose a numerical solution of the equations. 


\section{Numerical scheme}

In order to construct an appropriate numerical procedure, we first remark that the $\varphi$ functions are vanishing at infinity irrespective of the asymptotic behavior of the repair time density functions! Therefore, a numerical procedure to solve the equations in the region $(0, \infty) \times(0, \infty)$ may be converted into a numerical solution procedure in the truncated region $(0, L) \times(0, L)$, for some $L>0$, with prescribed boundary conditions $\varphi_{B}(L)=$ $\varphi_{C}(L)=\varphi_{D}(L, \cdot)=\varphi_{D}(\cdot, L)=0$. Let $\varphi_{B, i}:=\varphi_{B}\left(x_{i}\right), \varphi_{C, j}:=\varphi_{C}\left(y_{j}\right), \varphi_{D, i, j}:=\varphi_{D}\left(x_{i}, y_{i}\right)$, where $x_{i}:=i \Delta, y_{j}:=j \Delta, i=0, \ldots, N+1 ; j=0, \ldots, N+1 ; \Delta:=L / N$. We propose the following numerical scheme. Let $k$ be the iteration number. Given $\varphi_{D, i, N+1}^{k+1}=0, \varphi_{D, N+1, j}^{k+1}=0$, $\varphi_{B, N+1}^{k+1}=0, \varphi_{C, N+1}^{k+1}=0$, and the values of $\varphi_{B, i}^{k}$ and $\varphi_{C, j}^{k}$, we compute $\varphi_{D, i, j}^{k+1}$ by means of the two-point first-order approximation of (4.4), namely,

$$
\varphi_{D, i, j}^{k+1}=\frac{1}{2}\left(\varphi_{D, i, j+1}^{k+1}+\varphi_{D, i+1, j}^{k+1}\right)+\frac{\Delta}{2}\left(\lambda_{s} \varphi_{B, i}^{k} r_{s, j}+\lambda \varphi_{C, j}^{k} r_{i}\right)
$$

$i=N, N-1, \ldots, 0$ and $j=N, N-1, \ldots, 0$.

Next, we calculate $\varphi_{B, i}^{k+1}$ and $\varphi_{C, j}^{k+1}$ by means of the first-order approximations of (4.2) and (4.3) given by

$$
\begin{gathered}
\varphi_{B, i}^{k+1}=\frac{1}{\gamma_{B}}\left(\frac{\varphi_{B, i+1}^{k+1}}{\Delta}+\varphi_{D, i, 0}^{k+1}+\lambda r_{i}\right), \\
\varphi_{C, j}^{k+1}=\frac{1}{\gamma_{C}}\left(\frac{\varphi_{C, j+1}^{k+1}}{\Delta}+\varphi_{D, 0, j}^{k+1}\right),
\end{gathered}
$$

where $\gamma_{B}:=\lambda_{s}+1 / \Delta$ and $\gamma_{C}:=\lambda+1 / \Delta$. Finally, in order to satisfy (4.1) we use the normalizing procedure

$$
\begin{aligned}
\varphi_{C, j}^{k+1, \text { new }} & =\lambda \frac{\varphi_{C, j}^{k+1}}{\varphi_{C, 0}^{k+1}+\varphi_{B, 0}^{k+1}}, \\
\varphi_{B, i}^{k+1, \text { new }} & =\lambda \frac{\varphi_{B, i}^{k+1}}{\varphi_{C, 0}^{k+1}+\varphi_{B, 0}^{k+1}} .
\end{aligned}
$$

Remarks 5.1. Let $\varphi_{\Delta}$ denote a numerical solution obtained with the space-step $\Delta$. The relevant numerical error is then evaluated on a nested grid by $\varepsilon:=\left|\varphi_{\Delta}-\varphi_{\Delta / 2}\right|$. However, such an estimate is only accurate if $L$ is large enough. Roughly speaking, if $\max \left(r(x), r_{s}(x)\right)$ at $x=L$ is small, then (most likely) this particular $L$ is appropriate. However, such a "brutal force" approach may require a large number of grid points and is therefore rarely applicable. We illustrate the phenomenon by comparing the exact and the numerical solution in the most simple case, that is, let $R(x)=1-e^{-x}, R_{S}(y)=1-e^{-y}$. Then, $\varphi_{D}(x, y)=$ $l_{D} e^{-(x+y)}, \varphi_{C}(y)=l_{C} e^{-y}, \varphi_{B}(x)=l_{B} e^{-x}$, where $l_{D}:=\lambda_{s}(\lambda+1) \lambda /\left(\lambda_{s}+\lambda+2\right), l_{C}:=\lambda_{s} \lambda /\left(\lambda_{s}+\right.$ $\lambda+2), l_{B}:=\lambda(\lambda+2) /\left(\lambda_{s}+\lambda+2\right)$.

Figure 5.1 shows the numerical error

$$
\varepsilon_{M}:=\max \left\{\left|\varphi_{D}^{\text {exact }}-\varphi_{D}\right|,\left|\varphi_{C}^{\text {exact }}-\varphi_{C}\right|,\left|\varphi_{B}^{\text {exact }}-\varphi_{B}\right|\right\}
$$

versus the grid size for various $L$. 


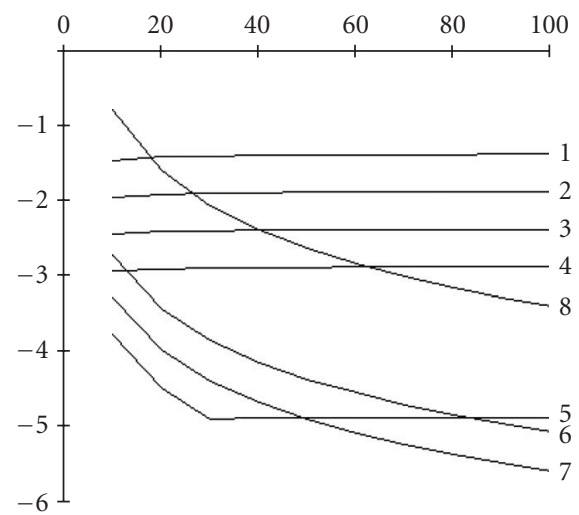

Figure 5.1. The horizontal axis denotes the logarithm of the numerical error, the vertical axis denotes the number of the grid points, (1) $L=0.4$; (2) $L=1.0$; (3) $L=1.5$; (4) $L=2$; (5) $L=4$; (6) $L=6$; (7) $L=10$; (8) $L=50$.

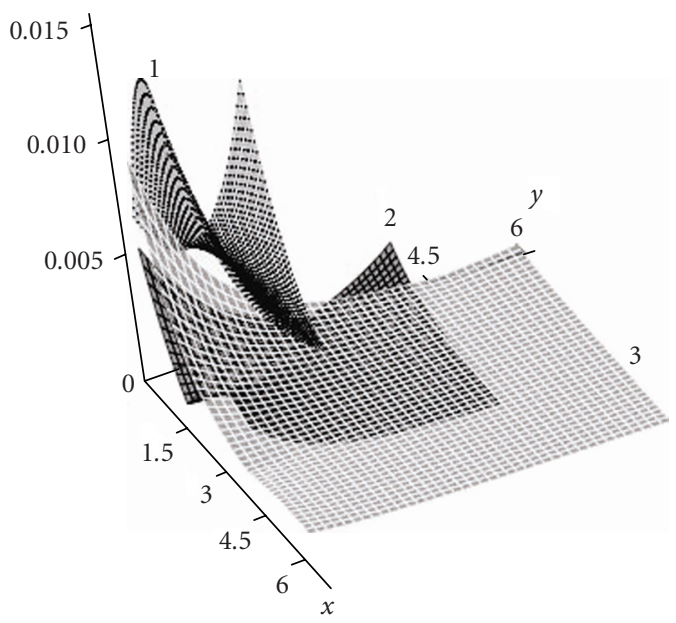

Figure 5.2. Spatial distribution of $\varepsilon_{D}$, (1) $L=1.5$; (2) $L=3$; (3) $L=6$.

Observe that, if $L$ is not large enough, $\varepsilon_{M}$ does not decrease as $\Delta$ decreases (see Figure 5.1). On the other hand, too large $L$ (consequently, too large $\Delta$ ) lead to large numerical errors. For instance, the error with $L=30$ is larger than $2.5 \cdot 10^{-2}$ for any $N \in[20,100]$, whereas the error with $L=4$ is less than $2.5 \cdot 10^{-2}$. There could be multiple options too. For instance, an error less than $2.5 \cdot 10^{-2}$ is achieved either with $L=4$, $N=15$, or $L=6, N=22$, or $L=10, N=38$.

Figure 5.2 shows a two-dimensional spatial distribution of the error $\varepsilon_{D}:=\left|\varphi_{D}^{\text {exact }}-\varphi_{D}\right|$ for various $L$. Clearly, $\varepsilon_{D}$ could be increasing near the origin as $L$ increases. However, the error decreases for large $x$ and $y$. 


\section{Trial-and-error procedure}

The complicated behavior of the numerical error requires an adaptive choice of $\Delta$ and $L$. Therefore, we introduce the subordinate errors $\varepsilon_{1}:=\left|\varphi_{\Delta, L}-\varphi_{\Delta, L / 2}\right|$ and $\varepsilon_{2}:=\mid \varphi_{\Delta, L}-$ $\varphi_{\Delta / 2, L} \mid$, where $\varepsilon_{1}$ characterizes the numerical error caused by truncation of the infinite region and $\varepsilon_{2}$ the numerical error related to the first-order approximants. In order to find the optimal pair $(L, \Delta)$, we first specify the required accuracy $\varepsilon$. Next, we propose the following trial-and-error procedure: we vary L until $\varepsilon_{1}<\varepsilon$ and then $\Delta$ until $\varepsilon_{2}<\varepsilon$. Finally, we introduce the following.

\section{Application. The Weibull-Gnedenko distribution}

We consider the particular but important case of Weibull-Gnedenko repair time distributions, that is, let $R(x)=1-e^{-x^{\beta_{1}}}, R_{S}(y)=1-e^{-y^{\beta_{2}}}$. Obviously, the optimal pair $(L, \Delta)$ depends on $\lambda, \lambda_{s}, \beta_{1}$, and $\beta_{2}$. We demonstrate the trial-and-error procedure applied to the particular case $\lambda=1, \lambda_{s}=0.1, \beta_{1}=2, \beta_{2}=3$. However, no restrictions are imposed on the analysis of $\mathscr{A}$ for arbitrary values of $\lambda, \lambda_{s}, \beta_{1}$ and $\beta_{2}$. Let $\varepsilon=10^{-3}$.

First, we vary $L$, as shown in Table 7.1, until $\varepsilon_{1}<\varepsilon$. Next, we vary $\Delta$, as shown in Table 7.2, until $\varepsilon_{2}<\varepsilon$. A spatial distribution of $\varepsilon_{1}$ and $\varepsilon_{2}$ is depicted in Figures 7.1 and 7.2 .

Table 7.1. The $L$ trials.

\begin{tabular}{cccc}
\hline$L$ & $N$ & $\Delta$ & $\varepsilon_{1}$ \\
\hline 3 & 40 & $3 / 40$ & $1.9 \cdot 10^{-2}$ \\
6 & 80 & $3 / 40$ & $7.4 \cdot 10^{-4}$ \\
\hline
\end{tabular}

Table 7.2. The $\Delta$ trials.

\begin{tabular}{cccc}
\hline$L$ & $N$ & $\Delta$ & $\varepsilon_{2}$ \\
\hline 6 & 80 & $3 / 40$ & $6.8 \cdot 10^{-3}$ \\
6 & 160 & $3 / 80$ & $3.3 \cdot 10^{-3}$ \\
6 & 320 & $3 / 160$ & $9.2 \cdot 10^{-4}$ \\
\hline
\end{tabular}

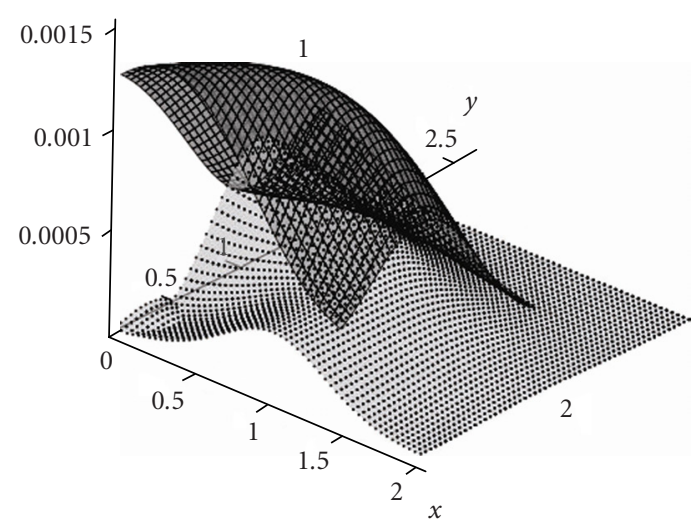

Figure 7.1. Spatial distribution of $\varepsilon_{1}$, (1) $L=3$; (2) $L=6$. 
82 Numerical availability of a priority system

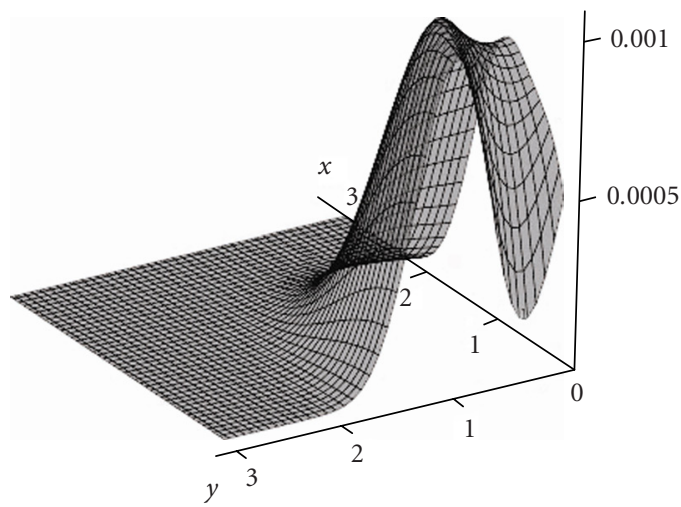

Figure 7.2. Spatial distribution of $\varepsilon_{2}$ for $N=320$.

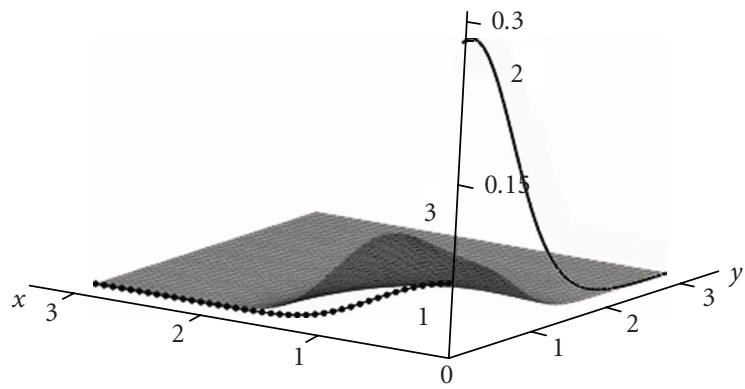

Figure 7.3. Numerically generated: (1) $p_{B}(x) / 1.5,(2) p_{C}(x),(3) p_{D}(x, y), \lambda_{s}=0.3 \lambda=1.0$. Note that $p_{B}$ is divided by 1.5 due to scaling.

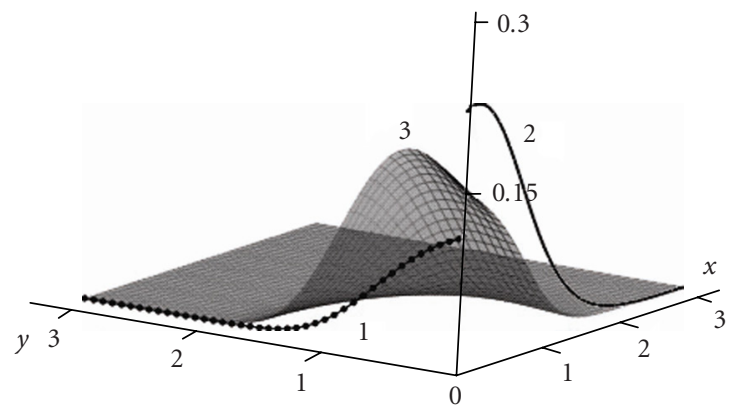

Figure 7.4. Numerically generated: (1) $p_{B}(x) / 1.5$, (2) $p_{C}(x),(3) p_{D}(x, y), \lambda_{s}=0.7 \lambda=1.0$. Note that $p_{B}$ is divided by 1.5 due to scaling.

Figure 7.3 displays $p_{B}(\cdot), p_{C}(\cdot)$, and $p_{D}(\cdot, \cdot)$ for $\lambda=1, \lambda_{s}=0.3$ and Figure 7.4 for $\lambda=$ $1, \lambda_{s}=0.5$. Figure 7.5 shows $p_{D}(x, y)$ for various $\lambda_{s}$. Let $\mathscr{A}_{\beta_{1}, \beta_{2}}\left(\lambda, \lambda_{s}\right)$ denote the long-run availability as a function of $\lambda$ and $\lambda_{s}$. 


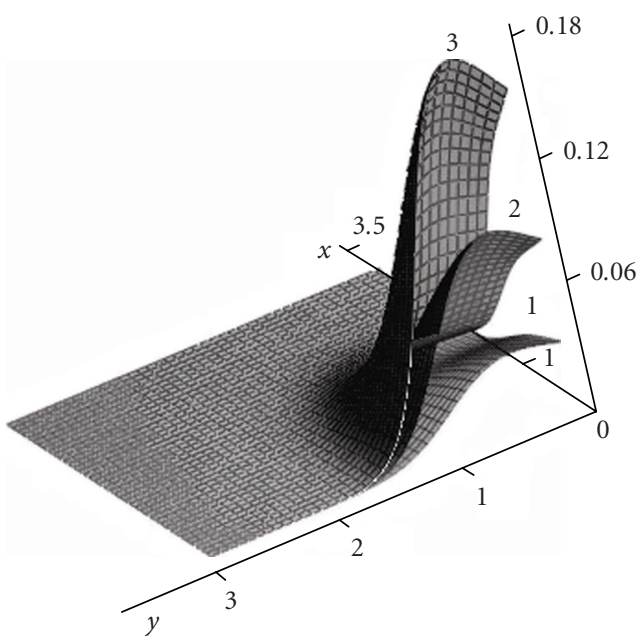

Figure 7.5. Numerically generated $p_{D}(x, y), \lambda=1.0,(1) \lambda_{s}=0.1,(2) \lambda_{s}=0.3$, (3) $\lambda_{s}=0.7$.

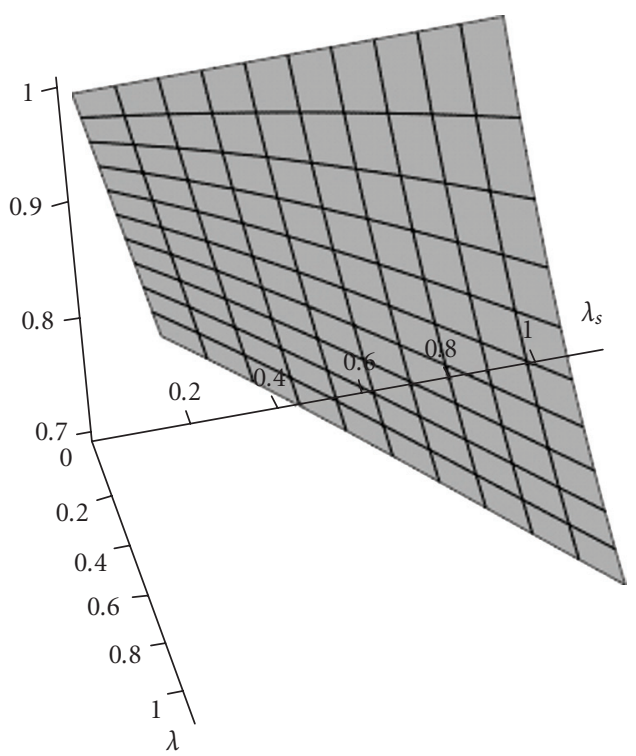

Figure 7.6. Numerically generated long-run availability.

Figure 7.6 shows that the long-run availability exhibits a nonlinear behavior for sufficiently large $\lambda$ and $\lambda_{s}$ (see also Table 7.3). Finally, Figure 7.7 displays the deviations $d_{1}:=\left|\mathscr{A}_{2,2}-\mathscr{A}_{2,4}\right|, d_{2}:=\left|\mathscr{A}_{2,2}-\mathscr{A}_{4,2}\right|, d_{3}:=\left|\mathscr{A}_{2,2}-\mathscr{A}_{4,4}\right|$. The plot reveals that $\mathscr{A}$ is fairly insensitive for $\beta$-variations. 
84 Numerical availability of a priority system

Table 7.3. Long-run availability $\mathscr{A}_{2,2}\left(\lambda, \lambda_{s}\right)$.

\begin{tabular}{c|cccccccccc}
\hline$\lambda / \lambda_{s}$ & 0.1 & 0.2 & 0.3 & 0.4 & 0.5 & 0.6 & 0.7 & 0.8 & 0.9 & 1.0 \\
\hline 0.1 & 0.9950 & 0.9895 & 0.9837 & 0.9775 & 0.9709 & 0.9640 & 0.9566 & 0.9489 & 0.9408 & 0.9324 \\
0.2 & 0.9908 & 0.9811 & 0.9706 & 0.9596 & 0.9480 & 0.9358 & 0.9231 & 0.9098 & 0.8962 & 0.8821 \\
0.3 & 0.9874 & 0.9740 & 0.9599 & 0.9450 & 0.9295 & 0.9133 & 0.8965 & 0.8792 & 0.8615 & 0.8434 \\
0.4 & 0.9845 & 0.9681 & 0.9509 & 0.9330 & 0.9143 & 0.8949 & 0.8750 & 0.8546 & 0.8338 & 0.8128 \\
0.5 & 0.9820 & 0.9631 & 0.9434 & 0.9229 & 0.9016 & 0.8797 & 0.8573 & 0.8345 & 0.8114 & 0.7882 \\
0.6 & 0.9798 & 0.9588 & 0.9369 & 0.9143 & 0.8909 & 0.8670 & 0.8426 & 0.8179 & 0.7930 & 0.7680 \\
0.7 & 0.9779 & 0.9550 & 0.9313 & 0.9069 & 0.8818 & 0.8562 & 0.8301 & 0.8039 & 0.7775 & 0.7512 \\
0.8 & 0.9762 & 0.9517 & 0.9265 & 0.9005 & 0.8740 & 0.8469 & 0.8195 & 0.7920 & 0.7644 & 0.7370 \\
0.9 & 0.9748 & 0.9488 & 0.9222 & 0.8949 & 0.8671 & 0.8389 & 0.8103 & 0.7817 & 0.7532 & 0.7249 \\
1.0 & 0.9734 & 0.9462 & 0.9184 & 0.8900 & 0.8611 & 0.8318 & 0.8024 & 0.7729 & 0.7435 & 0.7145 \\
\hline
\end{tabular}

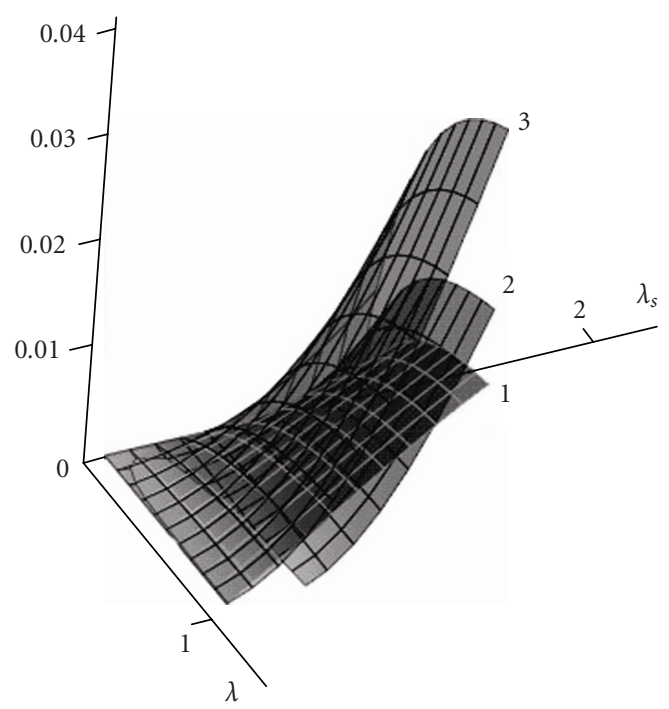

Figure 7.7. Spatial deviations $d_{i}, i=1,2,3$.

\section{Conclusion}

An effective statistical analysis of the T-system requires the solution of coupled Hokstadtype differential equations. Our numerical solution procedure, endowed with a simple and robust algorithm, allows to compute and to analyze the long-run availability for a general class of current repair time distributions with tangible engineering applications.

\section{References}

[1] J. R. Arora, Reliability of a two-unit priority standby redundant system with finite repair capability, IEEE Trans. Reliab. R-25 (1976), 205-207. 

, Reliability of several standby priority redundant systems, IEEE Trans. Reliab. R-30 (1981), 123-132.

[3] A. Birolini, Quality and Reliability of Technical Systems, Springer-Verlag, Berlin, 1994.

[4] J. A. Buzacott, Availability of priority redundant systems, IEEE Trans. Reliab. R-20 (1971), 60-63.

[5] B. B. Fawzi and A. G. Hawkes, Availability of a series system with replacement and repair, J. Appl. Probab. 27 (1990), no. 4, 873-887.

[6] I. B. Gertsbakh, Statistical Reliability Theory, Probability: Pure and Applied, vol. 4, Marcel Dekker, New York, 1989.

[7] B. Gnedenko and I. A. Ushakov, Probabilistic Reliability Engineering, John Wiley \& Sons, New York, 1995.

[8] L. R. Goel and S. K. Singh, Cost analysis of a two-unit priority standby system with imperfect switch, intermittent repair and arbitrary distributions, IEEE Trans. Reliab. R-35 (1986), 585586.

[9] R. Gupta and L. R. Goel, Profit analysis of a two-unit priority standby system with administrative delay in repair, Internat. J. Systems Sci. 20 (1998), 1703-1712.

[10] W. Li and D. H. Shi, Reliability analysis of a two-dissimilar-unit parallel redundant system with preemptive priority rule, Microelectron. Reliab. 33 (1993), no. 10, 1447-1453.

[11] H. Mine, Repair priority effect on the availability of a two-unit system, IEEE Trans. Reliab. R-28 (1979), 325-326.

[12] T. Nakagawa and S. Osaki, Stochastic behaviour of a two-unit priority standby redundant system with repair, Microelectron. Reliab. 14 (1975), no. 3, 309-313.

[13] S. Osaki, Reliability analysis of a two-unit standby redundant system with priority, Can. Oper. Res. Soc. J. 8 (1970), 60-62.

[14] H. Pham, Recent Advances in Reliability and Quality Engineering, World Scientific Publishing, Singapore, 2001.

[15] M. Shaked and J. G. Shanthikumar, Reliability and maintainability, Stochastic Models (D. P. Heyman and M. J. Sobel, eds.), Handbooks Oper. Res. Management Sci., vol. 2, NorthHolland, Amsterdam, 1990, pp. 653-713.

[16] D.-H. Shi and L. Liu, Availability analysis of a two-unit series system with a priority shut-off rule, Naval Res. Logist. 43 (1996), no. 7, 1009-1024.

[17] R. Subramanian and N. Ravichandran, Probabilistic analysis of a two-unit standby redundant system with finite repair capacity, Opsearch 17 (1980), no. 2-3, 71-80.

[18] _ Analysis of a two unit warm standby system, Opsearch 21 (1984), 23-29.

[19] K. Val'des Kastro, Optimal policies in a priority standby model, Vestnik Moskov. Univ. Ser. I Mat. Mekh. 41 (1986), no. 4, 10-15.

[20] E. J. Vanderperre, Reliability analysis of a two-unit cold standby redundant system subject to a priority rule, Cahiers Centre Études Rech. Opér. 31 (1989), no. 1-2, 159-166.

[21]_, Long-run availability of a two-unit standby system subjected to a priority rule, Bull. Belg. Math. Soc. Simon Stevin 7 (2000), no. 3, 355-364.

[22] E. J. Vanderperre, S. S. Makhanov, and S. Suchatvejapoom, Long-run availability of a repairable parallel system, Microelectron. Reliab. 37 (1997), no. 3, 525-527.

Edmond J. Vanderperre: Department of Quantitative Management, University of South Africa, P.O. Box 392, Pretoria 0003, South Africa

E-mail address: edmondvanderperre@hotmail.com

Stanislav S. Makhanov: Faculty of Information Technology, Sirindhorn International Institute of Technology, Thammasat University, P.O. Box 22, Patumthani 12121, Thailand

E-mail address: makhanov@siit.tu.ac.th 


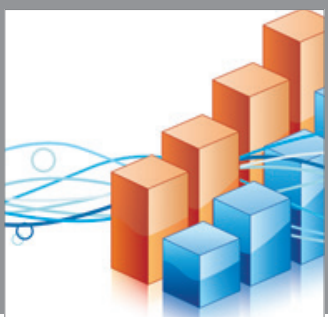

Advances in

Operations Research

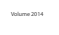

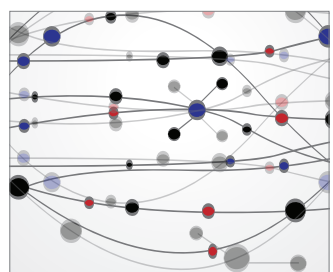

\section{The Scientific} World Journal
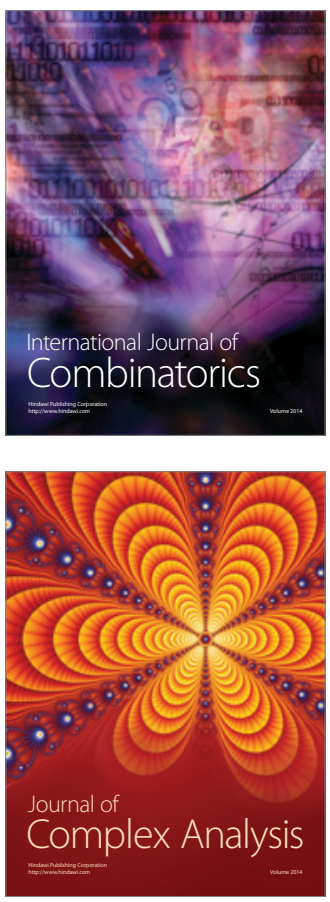

International Journal of

Mathematics and

Mathematical

Sciences
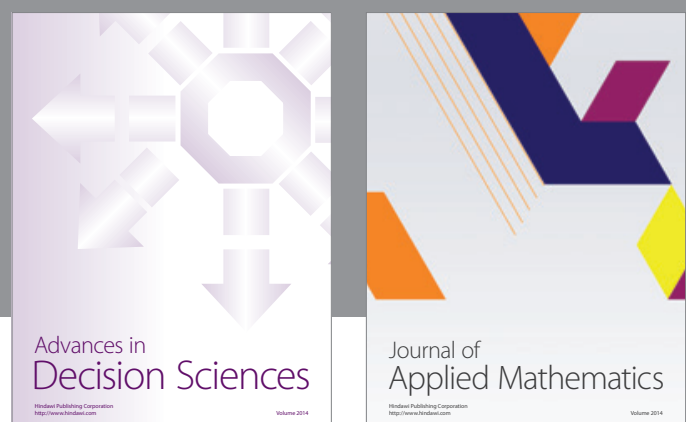

Journal of

Applied Mathematics
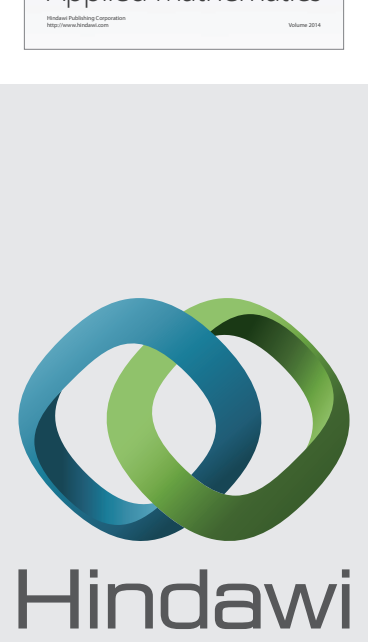

Submit your manuscripts at http://www.hindawi.com
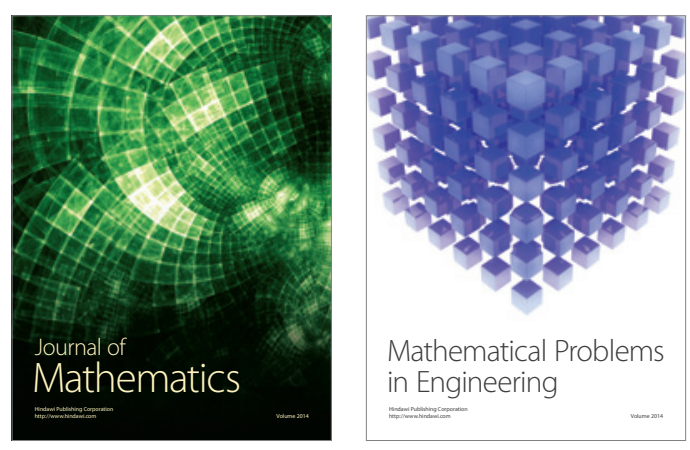

Mathematical Problems in Engineering
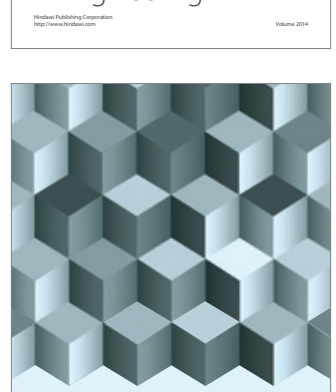

Journal of

Function Spaces
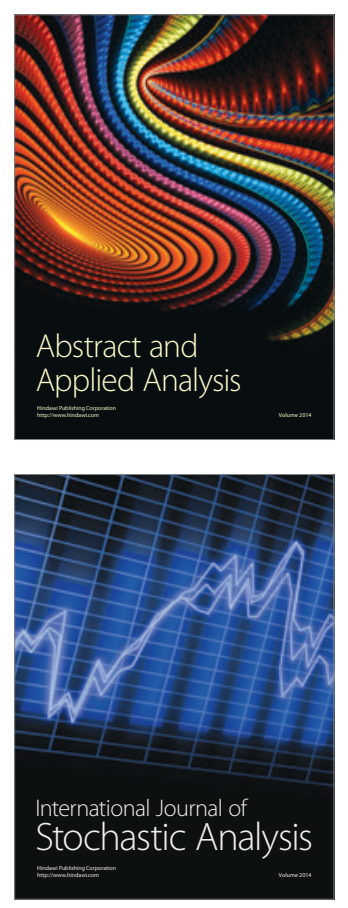

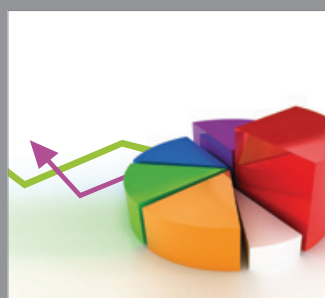

ournal of

Probability and Statistics

Promensencen
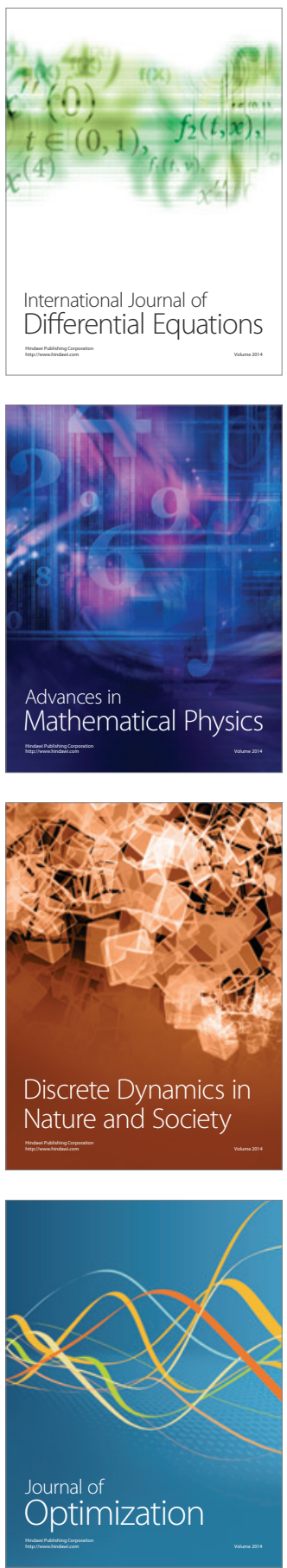